\title{
Characteristics of Gate-All-Around Twin Poly-Si Nanowire Thin-Film Transistors
}

\author{
Jeng-Tzong Sheu, Po-Chun Huang, Tzu-Shiun Sheu, Chen-Chia Chen, and Lu-An Chen
}

\begin{abstract}
We have investigated the characteristics of gate-allaround (GAA) twin polycrystalline-silicon nanowire (NW) thinfilm transistors (TFTs). The NW channel and surrounding gate imparted the GAA twin NW TFT with superior channel controllability. Moreover, the combination of the high surface-to-volume ratio of the $\mathrm{NW}$ and the split channel structure led to highly efficient $\mathrm{NH}_{3}$ plasma treatment, which reduced the effective grainboundary trap-state density. The GAA twin NW TFT exhibited greatly improved electrical performance, including a lower threshold voltage, a steeper subthreshold swing $(114 \mathrm{mV} / \mathrm{dec})$, a higher on/off current ratio $\left(>10^{8}\right)$, and a virtual absence of draininduced barrier lowering $(13 \mathrm{mV} / \mathrm{V})$.
\end{abstract}

Index Terms-Gate-all-around (GAA), nanowire (NW), plasma treatment, short-channel effects (SCEs), thin-film transistor (TFT).

\section{INTRODUCTION}

$\mathbf{P}$ OLYCRYSTALLINE-SILICON thin-film transistors (poly-Si TFTs) are attracting much attention for their use in active-matrix liquid crystal displays [1]. Increasing the performance of poly-Si TFTs has become one of the key challenges for system-on-chip applications. Unfortunately, traditional poly-Si TFTs suffer from serious short-channel effects (SCEs) when the device feature size is scaled down, due to the field-emission leakage current near the high-electric-field drain side and grain-boundary trap states in the channel [2][4]. Many crystallization techniques, including excimer-laser annealing (ELA), [5], [6] metal-induced lateral crystallization (MILC), [7], [8], and solid-phase crystallization (SPC) [9], have been used to improve the quality of poly-Si films, reduce the leakage current, enhance the carrier mobility, and suppress SCEs. Although ELA and MILC both result in poly-Si films exhibiting larger grain size, they add complexity to the fabrication process. Poly-Si films formed through SPC exhibit poorer performance because of the presence of a large number of randomly oriented grain boundaries [10]. A multiple-gated device has been proposed to enhance the gate controllability over the

Manuscript received August 13, 2008; revised November 4, 2008. First published December 31, 2008; current version published January 28, 2009. This work was supported in part by the National Science Council under Grant NSC-96-2221-E-009-213) and in part by the MOE-ATU Program in Taiwan. The review of this letter was arranged by Editor A. Chatterjee.

J.-T. Sheu, T.-S. Sheu, C.-C. Chen, and L.-A. Chen are with the Institute of Nanotechnology, National Chiao Tung University, Hsinchu 30050, Taiwan (e-mail: jtsheu@faculty.nctu.edu.tw; tzshin410@hotmail.com; s2323902@ ncnu.edu.tw; kamidochen@hotmail.com).

P.-C. Huang is with the Department of Materials Science and Engineering, National Chiao Tung University, Hsinchu 30050, Taiwan (e-mail: ginjel@ yahoo.com.tw).

Color versions of one or more of the figures in this letter are available online at http://ieeexplore.ieee.org.

Digital Object Identifier 10.1109/LED.2008.2009956 channel and thereby improve the device performance [6], [9], [11]. Although the gate-all-around (GAA) structure provides superior channel controllability, however, performance of the poly-Si TFT with the GAA structure still suffers from intrinsic defects in the poly-Si channel. In this letter, the performance of the GAA TFT was further improved by employing $\mathrm{NH}_{3}$ plasma treatment for the reduction of trap-state density. Only few research works discussed these considerations [6], [12], [13]. Furthermore, the spacer patterning technique was adopted for linewidth uniformity and controllability without using expensive advance lithographic tools [7], [9].

\section{DEVICE FABRICATION}

The 3- $\mu \mathrm{m}$-long twin poly-Si nanowire (NW) was fabricated using the spacer patterning technique [14]. Wafers (p-type, ca. $10^{15} / \mathrm{cm}^{3}$ ) were subjected to thermal oxidation to form a 400-nm $\mathrm{SiO}_{2}$ that served as the starting substrate. A $50-\mathrm{nm}$ thick undoped amorphous-Si $(\alpha-\mathrm{Si})$ layer was deposited at $550{ }^{\circ} \mathrm{C}$ using low-pressure chemical vapor deposition (LPCVD). SPC was performed at $600{ }^{\circ} \mathrm{C}$ for $24 \mathrm{~h}$ in a nitrogen ambient to turn the $\alpha$-Si into a polycrystalline-silicon structure. Next, a 100-nm-thick tetraethylorthosilicate (TEOS) oxide was deposited through LPCVD to serve as the dummy oxide layer. After patterning the dummy oxide layer using optical lithography, a $100-\mathrm{nm} \mathrm{SiN}_{x}$ film was deposited; subsequent reactive ion etching (RIE) formed the sidewall spacers that served as a hard mask in the following poly-Si etching process. By controlling the RIE conditions and the thickness of the $\mathrm{SiN}_{x}$ film, the feature size of the $\mathrm{SiN}_{x}$ units could be scaled down to the nanoscale without using any advanced photolithography techniques. Next, the TEOS dummy block was stripped off, and then, anisotropic RIE of poly-Si layer led to the formation of the twin poly-Si NW in which the wire width was predefined by the size of the $\mathrm{SiN}_{x}$ features. The poly-Si NW was then released from the thermal oxide through wet etching in $1: 100$ diluted $\mathrm{HF}$ solution by removing 70-80 $\mathrm{nm}$ of thermal $\mathrm{SiO}_{2}$, followed by sequential conformal deposition of 20-nm TEOS and 200-nm in situ $\mathrm{N}^{+}$polysilicon using LPCVD. The channel was surrounded by TEOS and $\mathrm{N}^{+}$polysilicon. After transferring the $2-\mu \mathrm{m}$ poly gate, self-aligned phosphorous ion implantation was performed at a dose of $5 \times 10^{15} \mathrm{~cm}^{-2}$, and then, the samples were activated through thermal annealing at $600{ }^{\circ} \mathrm{C}$ for $12 \mathrm{~h}$. A 300-nm-thick TEOS layer was deposited as the passivation layer using LPCVD. Finally, the contact hole was defined, and Al metallization was performed. All devices were sintered at $400{ }^{\circ} \mathrm{C}$ in a nitrogen ambient for $30 \mathrm{~min}$. To study the impact of grain-boundary defects, the samples were subjected to $\mathrm{NH}_{3}$ 


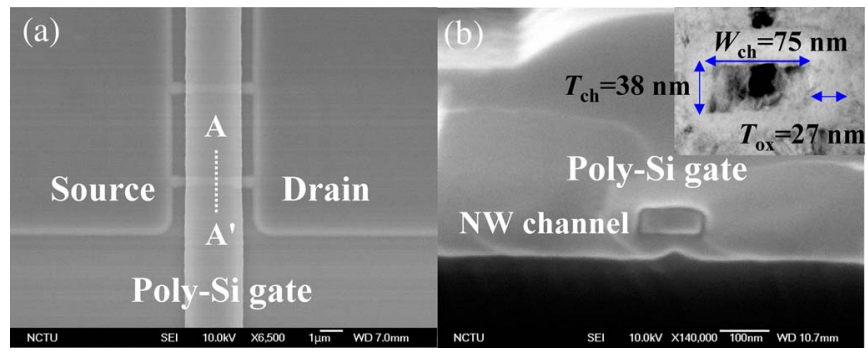

Fig. 1. (a) Top-view SEM photograph of the GAA twin poly-Si NW TFT. (b) SEM image of the cross-sectional view of the poly-Si NW which is surrounded by poly-Si gate electrode along the $\mathrm{AA}^{\prime}$ shown in (a). The inset of (b) shows a TEM photograph of the suspended NW $\left(W_{\mathrm{ch}}=75 \mathrm{~nm}\right.$ and $T_{\mathrm{ch}}=38 \mathrm{~nm}$ ) channel surrounded by $27-\mathrm{nm}$-thick TEOS and poly-Si gate.

TABLE I

Device Key Parameters of GAA Twin Poly-Si NW TFTs WITH $W_{\text {eff }} / L=0.452 \mu \mathrm{m} / 2 \mu \mathrm{m}$ AND PLANAR TFTs WITH $W / L=0.5 \mu \mathrm{m} / 2 \mu \mathrm{m}$. All PARAMETERS WerE EXTRACTED AT $V_{\mathrm{ds}}=0.5 \mathrm{~V}$, EXCEPT FOR THE ON/OFF CURRENT RATIO, WHICH WAS EXTRACTED AT $V_{\mathrm{ds}}=3.0 \mathrm{~V}$

\begin{tabular}{|c|c|c|c|c|}
\hline Structure $\left(\mathbf{W}_{\text {eff }} / \mathbf{L}\right)$ & $\mathbf{V}_{\text {th }}(\mathbf{V})$ & $\mathbf{I}_{\text {on }} / \mathbf{I}_{\text {off }}$ & $\begin{array}{c}\text { SS } \\
(\mathbf{m V} / \mathbf{d e c})\end{array}$ & $\begin{array}{c}\text { DIBL } \\
(\mathbf{m V} / \mathbf{V})\end{array}$ \\
\hline $\begin{array}{c}\mathrm{GAA}(0.452 \mu \mathrm{m} / 2 \\
\left.\mu \mathrm{m}, \mathrm{NH}_{3} \mathrm{~h}\right)\end{array}$ & $-0.38 \pm 0.177$ & $1.2 \times 10^{8}$ & 114 & 13 \\
\hline $\begin{array}{c}\mathrm{GAA}(0.452 \mu \mathrm{m} / 2 \\
\left.\mu \mathrm{m}, \mathrm{NH}_{3} 30 \mathrm{~min}\right)\end{array}$ & $0.73 \pm 0.317$ & $2.9 \times 10^{7}$ & 125 & 14 \\
\hline $\begin{array}{c}\mathrm{GAA}(0.452 \mu \mathrm{m} / 2 \\
\mu \mathrm{m}, \text { as fabricated) }\end{array}$ & $2.2 \pm 0.361$ & $2.5 \times 10^{6}$ & 466 & 24 \\
\hline $\begin{array}{c}\text { Planar }(0.5 \mu \mathrm{m} / 2 \\
\left.\mu \mathrm{m}, \mathrm{NH}_{3} 1 \mathrm{~h}\right)\end{array}$ & 0.9 & $2.1 \times 10^{7}$ & 402 & 15 \\
\hline $\begin{array}{c}\text { Planar }(0.5 \mu \mathrm{m} / 2 \\
\mu \mathrm{m}, \text { as fabricated })\end{array}$ & 1.12 & $3.3 \times 10^{6}$ & 611 & 96 \\
\hline
\end{tabular}

plasma treatment in a parallel-plate plasma reactor at a power density of $0.7 \mathrm{~W} / \mathrm{cm}^{2}$ at $300{ }^{\circ} \mathrm{C}$ for either $30 \mathrm{~min}$ or $1 \mathrm{~h}$.

\section{Results AND Discusion}

Fig. 1(a) shows a top-view scanning electron microscopy (SEM) image of the proposed GAA twin poly-Si NW TFT. Fig. 1(b) shows a cross-sectional SEM image viewed along the $\mathrm{AA}^{\prime}$ direction in Fig. 1(a). The poly-Si NW channel was surrounded by the $\mathrm{N}^{+}$poly-Si gate. The inset of Fig. 1(b) shows a transmission electron microscopy (TEM) image of the NW channel possessing the GAA structure. The proposed devices have a nominal channel length $(L)$ of $2 \mu \mathrm{m}$, a channel width $\left(W_{\mathrm{ch}}\right)$ of $75 \mathrm{~nm}$, and an effective channel width $\left(W_{\text {eff }}\right)$ of $452 \mathrm{~nm}\left[4 \times\left(W_{\mathrm{ch}}+T_{\mathrm{ch}}\right)\right]$. Because the aspect ratio $\left(T_{\mathrm{ch}} / W_{\mathrm{ch}}=0.5\right)$ is higher than that of a conventional planar TFT $\left(T_{\mathrm{ch}} / W_{\mathrm{ch}} \ll 0.1\right)$, we expected to observe improved device characteristics as a result of corner effects [15]. The additional electric field at the bottom corners and bottom of the channel in the GAA TFT enhances the performance so that the device exhibits a faster turn-on speed, a lower threshold voltage, and a smaller leakage current. Table I presents the key device parameters measured in GAA poly-Si NW TFTs and conventional planar gate poly-Si TFTs. GAA poly-Si NW TFTs exhibit a better device performance over conventional planar

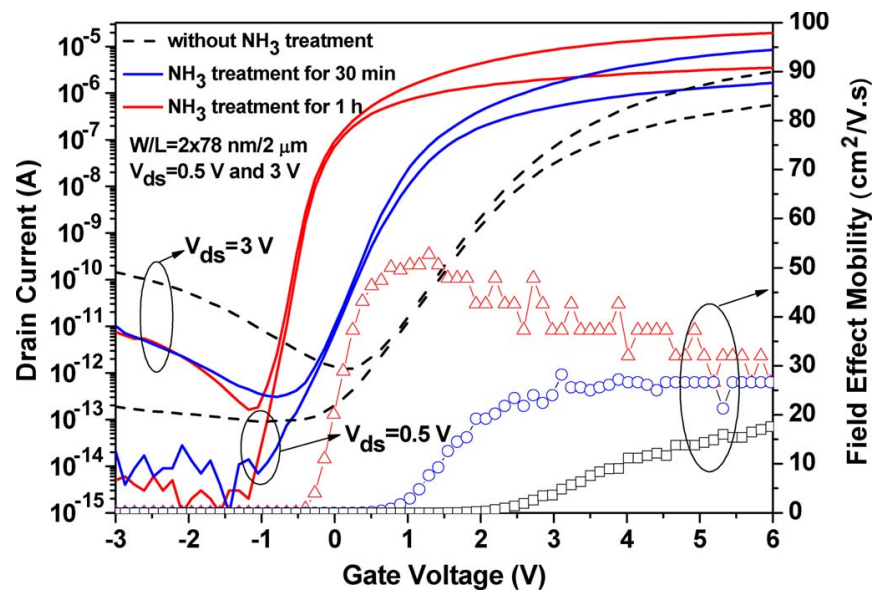

Fig. 2. Comparison of $I_{\mathrm{ds}}-V_{\mathrm{gs}}$ transfer characteristics and field-effect mobility of GAA twin poly-Si NW TFTs $(W / L=75 \mathrm{~nm} / 2 \mu \mathrm{m} \times 2)$ after passivation with $\mathrm{NH}_{3}$ plasma treatment for different periods.

poly-Si TFTs. In addition, after $\mathrm{NH}_{3}$ plasma treatment, the variations of the threshold voltage were minimized. Fig. 2 shows the normalized transfer characteristics of a typical GAA twin poly-Si NW TFT before and after $\mathrm{NH}_{3}$ plasma treatment at values of $V_{\mathrm{ds}}$ of 0.5 and $3 \mathrm{~V}$. The threshold voltage $\left(V_{\mathrm{th}}\right)$ is defined as the gate voltage required to achieve a normalized drain current of $I_{d}=\left(W_{\text {eff }} / L\right) \times 10^{-8}$ A at a value of $V_{\mathrm{ds}}$ of $0.5 \mathrm{~V}$. The subthreshold swing and field-effect mobility were extracted at a value of $V_{\mathrm{ds}}$ of $0.5 \mathrm{~V}$, whereas the on/off current ratio $\left(I_{\mathrm{on}} / I_{\mathrm{off}}\right)$ was extracted when $V_{\mathrm{ds}}$ was $3 \mathrm{~V}$, where $I_{\text {off }}$ is the minimum OFF-state current and $I_{\mathrm{on}}$ is the maximum ON-state current. Drain-induced barrier lowering (DIBL) is defined as $\Delta V_{\mathrm{gs}} / \Delta V_{\mathrm{ds}}$, where $I_{d}$ is $10^{-10} \mathrm{~A}$.

DIBL was well controlled to values less than $24 \mathrm{mV} / \mathrm{V}$, and it is improved further to $13 \mathrm{mV}$ when the devices were subjected to $\mathrm{NH}_{3}$ plasma treatment for $1 \mathrm{~h}$. SCEs were suppressed due to the channel being tightly controlled by the surrounding gate and because the grain-boundary-defect density was reduced after $\mathrm{NH}_{3}$ plasma treatment [13]. Additionally, $\mathrm{NH}_{3}$ plasma treatment reduced the leakage current significantly and provided the GAA twin poly-Si NW TFTs with a higher on-current. As a result, the GAA twin poly-Si NW TFTs exhibited a higher on/off current ratio $\left(>10^{8}\right)$ than that of the untreated samples $\left(>10^{6}\right)$. Similarly, the threshold voltage and subthreshold swing $(-0.38 \mathrm{~V}$ and $114 \mathrm{mV} / \mathrm{dec}$, respectively) were superior to those $(2.2 \mathrm{~V}$ and $466 \mathrm{mV} / \mathrm{dec})$ of the untreated device. Moreover, the maximum field-effect mobility of the GAA twin poly-Si NW TFTs increased dramatically from 23.4 to $52.7 \mathrm{~cm}^{2} / \mathrm{V} \cdot \mathrm{s}$. No channel doping process during self-aligned $\mathrm{N}^{+}$S/D implantation resulted in negative value of the threshold voltage. This problem can be solved by employing metal or metal silicide gates that have an appropriate work function [16].

To verify the reduction of grain-boundary defects in the GAA twin poly-Si NW TFTs after $\mathrm{NH}_{3}$ plasma treatment, we extracted the effective trap-state density $\left(N_{t}\right)$ from the slope of the plot of $\ln \left(I_{d} / V_{\mathrm{gs}}\right)$ versus $\left(1 / V_{g}\right)$ [3]. The GAA twin poly-Si NW TFTs had been subjected to $1 \mathrm{~h}$ of $\mathrm{NH}_{3}$ plasma treatment, revealing a reduction in the effective trap-state density $\left(N_{t}=\right.$ ca. $4.4 \times 10^{11}$ ) relative to that of the untreated GAA twin 


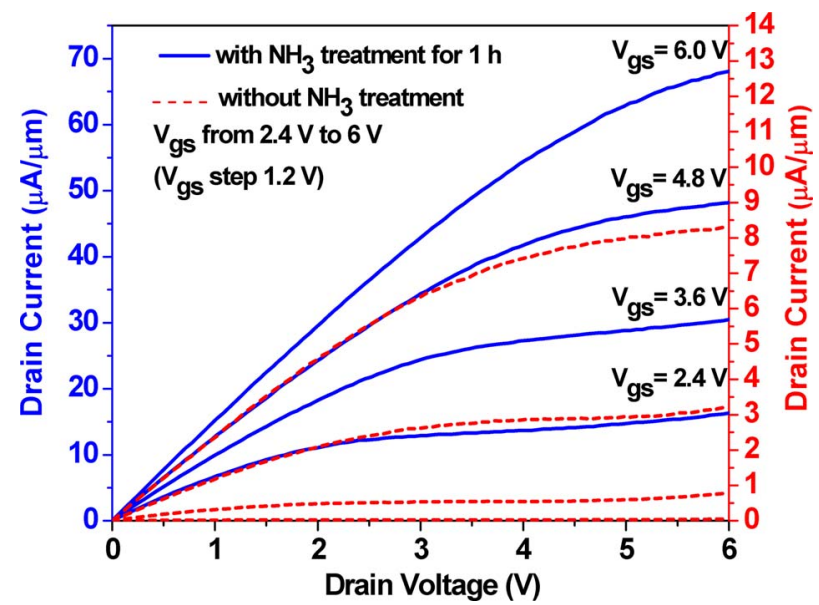

Fig. 3. Output $I_{\mathrm{ds}}-V_{\mathrm{ds}}$ characteristics of a GAA twin poly-Si NW TFT subjected to $\mathrm{NH}_{3}$ plasma treatment for $1 \mathrm{~h}$. Kink effect is suppressed by both GAA structure and $\mathrm{NH}_{3}$ plasma treatment.

poly-Si NW TFTs $\left(N_{t}=\right.$ ca. $\left.3.15 \times 10^{12}\right)$. We suspect that radicals diffused mainly through the gate oxide passivated the trap states in the channel during the $\mathrm{NH}_{3}$ plasma treatment process, and hence, the grain-boundary barrier potentials were further reduced [17]. Moreover, the high surface-to-volume ratio of the NWs and the split channel structure led to the higher efficiency of $\mathrm{NH}_{3}$-plasma-treated devices relative to that of conventional TFT devices (as shown in Table I for devices that had been subjected to 1-h plasma treatment).

Fig. 3 shows output characteristics typical for a GAA twin poly-Si NW TFT after $\mathrm{NH}_{3}$ plasma treatment for $1 \mathrm{~h}$. The drain saturation current was $12.2 \mu \mathrm{A} / \mu \mathrm{m}$ at values of $V_{\mathrm{ds}}$ and $V_{\mathrm{gs}}$ of $2.4 \mathrm{~V}(0.024 \mu \mathrm{A} / \mu \mathrm{m}$ for a device without plasma treatment). We suspect that performance characteristics could be improved further by optimizing the contact resistance through plug implantation or by using a metal silicide as source/drain regions. In the absence of a lightly doped drain, our proposed device structure features a steeper subthreshold slope, a greater on/off current ratio, and suppression of SCEs. As more and more functions are integrated on a flat-panel system, SCEs will make the power consumption problem for TFT applications like driving circuits in the active-matrix liquid crystal display more challenging. The proposed device exhibiting a low turnon voltage, a very low leakage current, and a high switching speed is highly promising for future applications.

\section{CONCLUSION}

In summary, we have characterized high-performance GAA twin poly-Si NW TFTs subjected to $\mathrm{NH}^{3}$ plasma treatment for $1 \mathrm{~h}$. The combination of the excellent gate controllability of the GAA structure and the $\mathrm{NH}_{3}$ plasma treatment process resulted in TFTs that were immune to both grain-boundary effects and SCEs. GAA twin poly-Si NW TFTs subjected to $\mathrm{NH}_{3}$ plasma treatment for $1 \mathrm{~h}$ exhibit a low threshold voltage $\left(V_{\mathrm{th}}=-0.38 \mathrm{~V}\right)$, a small subthreshold swing $(114 \mathrm{mV} / \mathrm{dec})$, a high on/off current ratio $\left(>10^{8}\right)$, and a virtual absence of DIBL. From the point of view of device integration, the wet etching process is the only process added in the fabrication of GAA NW poly-Si TFTs. We suspect that our proposed GAA twin poly-Si NW TFTs will be suitable for applications in lowvoltage $(<3.5 \mathrm{~V})$ circuit operations, high-performance driver circuits, and switching devices.

\section{ACKNOWLEDGMENT}

The authors would like to thank the National Nano Device Laboratories for its support in facility utilization.

\section{REFERENCES}

[1] T. Aoyama, K. Ogawa, Y. Mochizuki, and N. Konishi, "Inverse staggered poly-Si and amorphous Si double structure TFTs for LCD panels with peripheral driver circuits integration," IEEE Trans. Electron Devices, vol. 43, no. 5, pp. 701-705, May 1996.

[2] K. R. Olasupo and M. K. Hatalis, "Leakage current mechanism in submicron polysilicon thin-film transistors," IEEE Trans. Electron Devices, vol. 43, no. 8, pp. 1218-1223, Aug. 1996.

[3] J. Levinson, F. R. Shepherd, P. J. Scanlon, W. D. Westwood, G. Este, and M. Rider, "Conductivity behavior in polycrystalline semiconductor thin film transistors," J. Appl. Phys., vol. 53, no. 2, pp. 1193-1202, Feb. 1982.

[4] N. Yamauchi, J. J. Hajjar, and R. Reif, "Drastically improved performance in poly-Si TFTs with channel dimensions comparable to grain size," in IEDM Tech. Dig., 1989, pp. 353-356.

[5] H. Yin, W. Xianyu, H. Cho, X. Zhang, J. Jung, D. Kim, H. Lim, K. Park, J. Kim, J. Kwon, and T. Noguchi, "Advanced poly-Si TFT with fin-like channels by ELA," IEEE Electron Device Lett., vol. 27, no. 5, pp. 357359, May 2006.

[6] H. Yin, W. Xianyu, A. Tikhonovsky, and Y. S. Park, "Scalable 3-D finlike poly-Si TFT and Its nonvolatile memory application," IEEE Trans. Electron Devices, vol. 55, no. 2, pp. 578-584, Feb. 2008.

[7] C. W. Chang, S. F. Chen, C. L. Chang, C. K. Deng, J. J. Huang, and T. F. Lei, "High-performance nanowire TFTs with metal-induced lateral crystallized poly-Si channels," IEEE Electron Device Lett., vol. 29, no. 5, pp. 474-476, May 2008.

[8] Y. C. Wu, T. C. Chan, P. T. Liu, Y. C. Wu, C. W. Chou, C. H. Tu, J. C. Lou, and C. Y. Chang, "Mobility enhancement of polycrystalline-Si thin-film transistors using nanowire channels by pattern-dependent metal-induced lateral crystallization," Appl. Phys. Lett., vol. 87, no. 14, pp. 143504 143 506, Sep. 2005.

[9] C. J. Su, H. C. Lin, H. H. Tsai, H. H. Hsu, T. M. Wang, T. Y. Huang, and W. X. Ni, "Operations of poly-Si nanowire thin-film transistors with a multiple-gated configuration," Nanotechnology, vol. 18, no. 21, pp. 215 205-215211, May 2007.

[10] T. Uchida, "Present and future trend of electron device technology in flat panel display," in IEDM Tech. Dig., 1991, pp. 5-10.

[11] C. C. Tsai, Y. J. Lee, J. L. Wang, K. F. Wei, I. C. Lee, C. C. Chen, and H. C. Cheng, "High-performance top and bottom double-gate lowtemperature poly-silicon thin film transistors fabricated by excimer laser crystallization," Solid State Electron., vol. 52, no. 3, pp. 365-371, Mar. 2008.

[12] Y. C. Wu, T. C. Chang, C. W. Chou, Y. C. Wu, P. T. Liu, C. H. Tu, J. C. Lou, and C. Y. Chang, "Effects of channel width and $\mathrm{NH}_{3}$ plasma passivation on electrical characteristics of polysilicon thin-film transistors by pattern-dependent metal-induced lateral crystallization," J. Electrochem. Soc., vol. 152, no. 7, pp. G545-G549, Jun. 2005.

[13] H. C. Cheng, F. S. Wang, and C. Y. Huang, "Effects of $\mathrm{NH}_{3}$ plasma passivation on n-channel polycrystalline silicon thin-film transistors," IEEE Trans. Electron Devices, vol. 44, no. 1, pp. 64-68, Jan. 1997.

[14] Y. K. Choi, T. J. King, and C. Hu, "Spacer FinFET: Nanoscale doublegate CMOS technology for the terabit era," Solid State Electron., vol. 46, no. 10, pp. 1595-1601, Oct. 2002.

[15] J. W. Yang and J. G. Fossum, "On the feasibility of nanoscale triplegate CMOS transistors," IEEE Trans. Electron Devices, vol. 52, no. 6, pp. 1159-1164, Jun. 2005.

[16] J. Kedzierski, E. Nowak, T. Kanarsky, Y. Zhang, D. Boyd, R. Carruthers, C. Cabral, R. Amos, C. Lavoie, R. Roy, J. Newbury, E. Sullivan, J. Benedict, P. Saunders, K. Wong, D. Canaperi, M. Krishnan, K. L. Lee, B. A. Rainey, D. Fried, P. Cottrell, H. S. P. Wong, M. Ieong, and W. Haensch, "Metal-gate FinFET and fully-depleted SOI devices using total gate silicidation," in IEDM Tech. Dig., 2002, pp. 247-250.

[17] W. B. Jackson, N. M. Johnson, C. C. Tsai, I.-W. Wu, A. Chiang, and D. Smith, "Hydrogen diffusion in polycrystalline silicon thin films," Appl. Phys. Lett., vol. 61, no. 14, pp. 1670-1672, Oct. 1992. 